\title{
Out of Sight, Out of Mind, Right? Not in COVID-19 Shock or Anaerobic and Exhaustive Shock versus Septic Shock Dilemma That Means to Live or Die. Emergency Attention and a Necessity of Trials
}

\author{
Luiz Gonzaga Francisco de Assis Barros D’Elia Zanella ${ }^{1,2,3}$ \\ ${ }^{1}$ Infectious Diseases Department (MI-HSPE), Hospital do Servidor Público do Estado de São Paulo, São Paulo, Brazil \\ ${ }^{2} \mathrm{PhD}$ Program in Allergy and Immunopathology, School of Medicine, University of São Paulo, São Paulo, Brazil \\ ${ }^{3}$ Emergency and Urgency Department, University Hospital of University of São Paulo, Butantã, São Paulo, Brazil \\ Email: luiz.zanella@hc.fm.usp.br
}

How to cite this paper: D'Elia Zanella, L.G.F. de A.B. (2022) Out of Sight, Out of Mind, Right? Not in COVID-19 Shock or Anaerobic and Exhaustive Shock versus Septic Shock Dilemma That Means to Live or Die. Emergency Attention and a Necessity of Trials. Open Journal of Emergency Medicine, 10, 19-47. https://doi.org/10.4236/ojem.2022.101003

Received: December 12, 2021

Accepted: February 21, 2022

Published: February 24, 2022

Copyright $\odot 2022$ by author(s) and Scientific Research Publishing Inc. This work is licensed under the Creative Commons Attribution International License (CC BY 4.0).

http://creativecommons.org/licenses/by/4.0/ (c) (i) Open Access

\begin{abstract}
Background: COVID-19 brought challenges that did not end after a two-year pandemic. From more straightforward changes in habits to studying to understand the enigmatic parasite-host relationship, we can better manage the patient infected with SARS-CoV-2 even with a vaccine full of doubts and antivirals that do not correctly cover the viral period. SARS-CoV-2 brought the chronic inflammation now called "The Long COVID-19 Syndrome" (LCS), something still little talked about, but we already see deaths due to non-identification of this inflammatory syndrome that can lead to shock. Theory: LCS Shock is due to a long period of metabolic stress, reflecting the shift from inflammation to oxidative stress and innate immunity, and does not respond to antimicrobials, as its main component is inflammatory, although there may be conjoined bacterial translocation. Thus, we are losing patients to a new syndrome confused with sepsis and septic shock. While septic shock (SS) responds to antimicrobials, Inflammatory Shock (ISc) does not respond to antimicrobials alone, requiring high doses of corticosteroids. Review: This study shows that we need to differentiate SS and ISC, as the treatment is different. The review shows that Lactate, LDH and the presence of new/recent cardiac changes and bradycardia in the face of a status where there should be tachycardia as the usual response can differ ISC from SS. Maybe the main responsible for high LDH is Warburg Effect. Conclusion: We have a dilemma that requires clinical studies that routinely match high doses of corticosteroids (until there is something better to be done) and bring
\end{abstract}


laboratory and imaging differences to diagnose SS vs ISc better.

\section{Keywords}

Acute Respiratory Distress Syndrome, Cardiovascular System, Critical Care, COVID-19, Lactate

\section{Introduction}

Children present with post-COVID-19 Inflammatory Syndrome and are treated with corticosteroids and immunoglobulin. Adults also have post-COVID-19 Inflammatory Syndrome and are untreated, resulting in death. Children have post-COVID-19 Kawasaki vasculitis. Many adults too, but these vasculitides in adults are forgotten, resulting in death. It is no joke. It is Tragedy.

LCS by post-SARS-CoV-2 infection is a period of change in energy production metabolism (ATP) via oxidative stress and inflammation, whose chronicity promotes exhaustion of the immune system, tolerance, and systemic symptoms with the possibility of thrombosis. This review shows that it may be possible to differentiate classic septic shock from COVID-19 inflammatory shock. Currently, there is little literature on the subject. There is a need to organize clinical studies to investigate differentiating parameters between Septic Shock and Shock in LCS. In the absence of literature indicating a specific guide for the management of patients with COVID inflammatory shock, this article proposes, based on the literature and on the author's experience, that we pay attention to some details that can help in the fine and difficult management in the face of a new syndrome [1] [2].

In the appendix there is a proposal for criteria for differential diagnosis between shock by COVID-19 and shock septic, based on the author's review and experience.

\section{Methods}

\section{Inclusion Criteria for Articles}

The review was performed by searching for scientific articles on the PubMed platform using the descriptors "COVID-19" and "SHOCK". A total of 4007 articles were found between the years 2019 and 2021. It was established that the first 50 articles would be evaluated, and those that addressed multisystem inflammation in children would be discarded. Articles must be written in English. The evaluation criteria followed what is proposed by the Task Force of Academic Medicine and the GEA-RIME Committee APPENDIX 1, Academic Medicine: September 2001 - Volume 76 - Issue 9 - p 958-959.

Google Scholar was used to complement the study. More than 23 articles were added, totaling and articles. Most articles found addressed chronic inflammation in children. For populations over 18 years of age, there is still a rarity of publica- 
tions addressing the post-COVID-19 chronic inflammatory syndrome, with most of the data referring to the patient COVID-19 in acute disease. After being excluded, a total of 41 articles were used as references.

\section{Results}

\subsection{Kynurenine Pathway in COVID-19 and the Oxidative Stress/Innate Immunity Signatures, Obesity, and Immune Senescence}

The Kynurenine pathway is initiated by conversion of L-tryptophan (Try), by either of the enzymes tryptophan-2,3-dioxygenase or indoleamine 2,3-dioxygenase each forming formyl-kynurenine, which is then further degraded to kynurenine, the precursor of several bioactive compounds, including kynurenic acid, quinolinic acid, picolinic acid, and 3-hydroxyanthranilic acid. The pathway is responsible for over $90 \%$ of tryptophan metabolism in the periphery.

Kynureninase is a pyridoxal phosphate-dependent enzyme inhibited by estrogen and metabolites, with both vitamin B6 deficiency and inhibition. There is an increase in Kyn, HK, XA, and HaA and a decrease in KA and AA because of the simulation of a general vitamin B-6 deficiency in which the activities of the enzymes were not completely reduced [3] [4].

Naive CD4+T cells can differentiate into T helper (Th) cells, Th1, Th2, and Th17 lineages and regulatory $\mathrm{T}$ (Treg or $\mathrm{CD} 3+\mathrm{CD} 4+\mathrm{FOXP} 3+)$ cells. -6 and transforming growth factor $\beta$ (TGF $\beta$ ) is considered critical for the differentiation into Th17 cells. IL-21 and IL-23 pathways are involved in IL-6-programmed Th-17 cell differentiation. Various cytokines regulate th 17 differentiation. TGF- $\beta$ and IL- 6 induced th17 differentiation in mice, and IL- $1 \beta$ but not TGF- $\beta$ has been shown to participate in the development of Th17 cells together with IL-6 in humans [5] [6] [7] [8].

The development of Th17 cells is negatively regulated by IFN- $\gamma$, IL-27, and IL-2, the signals of which are dependent on Stat1 (IFN- $\gamma$ and IL-27) and Stat5 (IL-2), respectively. The orphan nuclear receptors, retinoid-related orphan receptor $\gamma(\operatorname{ROR} \gamma)$ and $\mathrm{ROR} \alpha$ have been identified as the key transcription factors that determine the differentiation of Th17 lineage. AhR is involved in the differentiation of Th17 cells by regulating Stat 1 activation, which suppresses Th17 cell differentiation under Th17-polarizing conditions (Figure 2).

Cellular changes in adipose tissue in young and aged mice. Young adipose tissue is highly enriched with iNKT cells, which support M2 macrophage and Treg expansion by production of IL-10 and IL-2, respectively. As mice age, iNKT cells are gradually replaced by a population of IL-17A-secreting $\gamma \delta$ T cells that support Tregs by a stromal cell-IL-33 axis. Lower figure. Nonredundant actions of iNKT and $\gamma \delta \mathrm{T}$ cells promote adipose tissue homeostasis. Top panel, Adipose iNKT cells expand Tregs via production of IL-2 and M2 macrophages via production of IL-10. Furthermore, activation of adipose iNKT cells induces weight loss and thermogenesis through an FGF21-dependent mechanism. Bottom panel, IL-17A-producing $\gamma \delta$ T cells in adipose tissue drive Treg expansion 
by inducing stromal cells to produce IL-33. Additionally, a $\gamma \delta$ IL-17A axis in adipose tissue is critical for maintenance of body temperature at thermoneutrality and upon cold challenge. iNKT cells and $\gamma \delta$ cells, two innate T lymphocytes, have emerged as key regulators of immune homeostasis in visceral adipose tissue, as well as rheostats controlling body temperature in response to environmental fluctuations in sc and brown adipose depots (Figure 2). iNKT cells control adipose Tregs and macrophage frequencies through IL-2 and IL-10 production, respectively, whereas $\mathrm{T}$ cells influence stromal cell expression of IL-33 through IL-17A production, and this in turn affects ST2+ Treg numbers in visceral adipose tissue. By producing different sets of cytokines, iNKT and T cells are both able to support adipose Tregs at adolescence and with age, respectively. Note that some studies found that adipose iNKT cells drive proinflammatory, pathogenic immune responses during obesity. These differences have not been reconciled but identifying the mechanisms behind them may reveal further insights into iNKT cell biology and how tissue-specific cues can drive different cell phenotypes [3] [4] [9] [10] [11] [12] [13].

\subsection{The Long COVID-19 Syndrome (in Adults)}

About $5 \%$ and $10 \%$ of patients infected with coronavirus, two severe acute respiratory syndromes (SARS-CoV-2) require admission to the Intensive Care Unit (ICU), with up to $67 \%$ developing shock, which was implicated as the leading cause of death in 7\% of coronavirus disease cases in 2019 (COVID-19) and contributed to an additional 33\%. All four types of shock-distributive, cardiogenic, obstructive, and hypovolemic shock-have been observed in patients with COVID-19 [1] [2] [5].

These data presented are related to patients in the acute phase of the disease. However, there are no data available regarding inflammatory shock in patients who developed CSF after hospital discharge.

LCS has been gradually identified as a different condition from sepsis but is still closely related to the classic management of septic shock. It is the closest reference for management, considering the similarities of these two nosological entities. However, the similarity has been a brake on the decision of an adequate treatment, which is based on the inflammatory blockade using high doses of corticosteroids [14] [15].

The presence of cardiac damage seems to be something prevalent even considering young patients who developed CSF with shock [2] [16] [17] [18] [19].

New-onset biventricular failure and vasoplegia progressed to shock with multiorgan dysfunction. All patients had a hyperinflammatory profile associated with vasoplegia and heart failure. Moreover, lymphopenia (and potentially high SARS-CoV-2 Ab titters) could be an indicator of a dysregulated immune system, augmenting the inflammatory cascade and tissue injury. Although SARS-CoV-2 Abs are presumed to have protective and neutralizing properties, the elevated levels of $\mathrm{Ab}$ could have played a pathogenic role in the observed hyperinflam- 
matory response. In SARS-CoV-2, non-neutralizing antibodies could cause an inflammatory cascade and enhance viral entry via an antibody-dependent mechanism enhancement. Alternatively, these cases may represent a new phenomenon in analogous adults to those recently described in the paediatric population. Several patients did have nonspecific rashes and other overlapping features though none had clinical vasculitis on coronary angiograms, and some patients had mild nonspecific late gadolinium enhancement on cardiac magnetic resonance imaging (Figure 1 and Figure 2).

The cause of LCS is multifactorial, but with increasing evidence focusing on acute Tryptophan and Phenylalanine deficiency due to ACE-2 internalization, it has brought light to the dark tunnel dug by SARS-CoV-2. This virus promotes metabolic changes in the body and damage to the organs where it passes during the acute phase of infection. These metabolic changes seem to be related to enzymatic mechanisms related to intracellular aerobic respiration. The shift from the Metabolic Try axis to the production of Kynurenine at the expense of the production of serotonin (5-HT) and Vitamin B3 (NAD/NADH+) seems to be

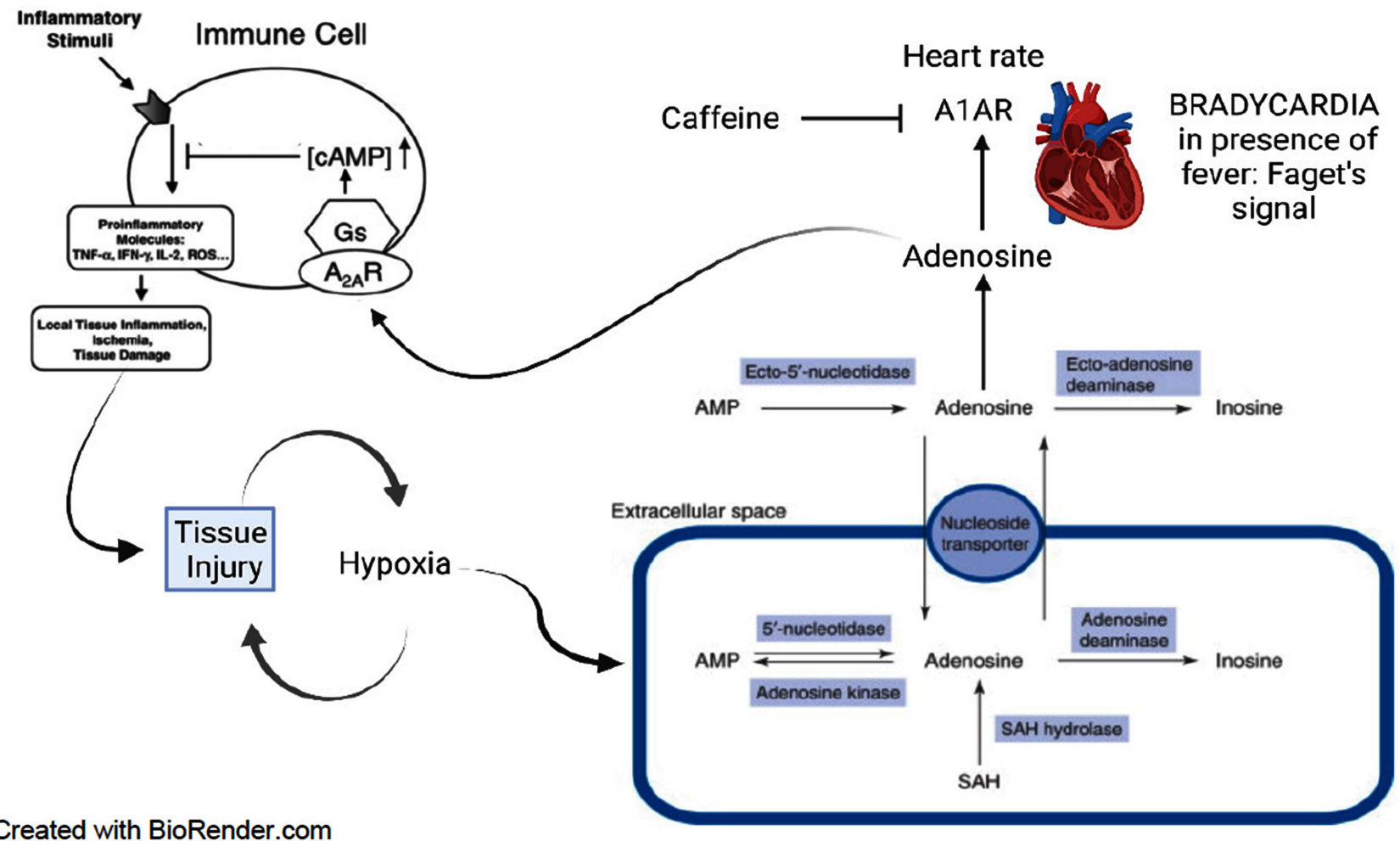

Figure 1. Faget's sign. Faget's sign is bradycardia concerning fever when the usual thing is tachycardia due to temperature elevations. It so happens that the pathophysiology behind this phenomenon is still controversial. Given the studies in COVID-19, the possibility arises that this phenomenon is related to the production of Adenosine (ADA) in the face of severe hypoxia that occurs in SARS-COV-2 infection. Adenosine plays an essential role against cell damage, has an anti-inflammatory/immunosuppressive role in addition to causing psychiatric phenomena that are very prevalent in COVID-19. However, this adaptation to cellular damage can become a sign of physical and metabolic exhaustion in the face of such intense inflammation resulting in Bradycardia with a risk of cardiorespiratory arrest due to the amount of serum adenosine and hypoxemia resulting from COVID-19. This situation also appears in other diseases that progress with severity and tissue hypoxia due to disseminated intravascular coagulation, causing cell damage due to lack of tissue oxygen. 


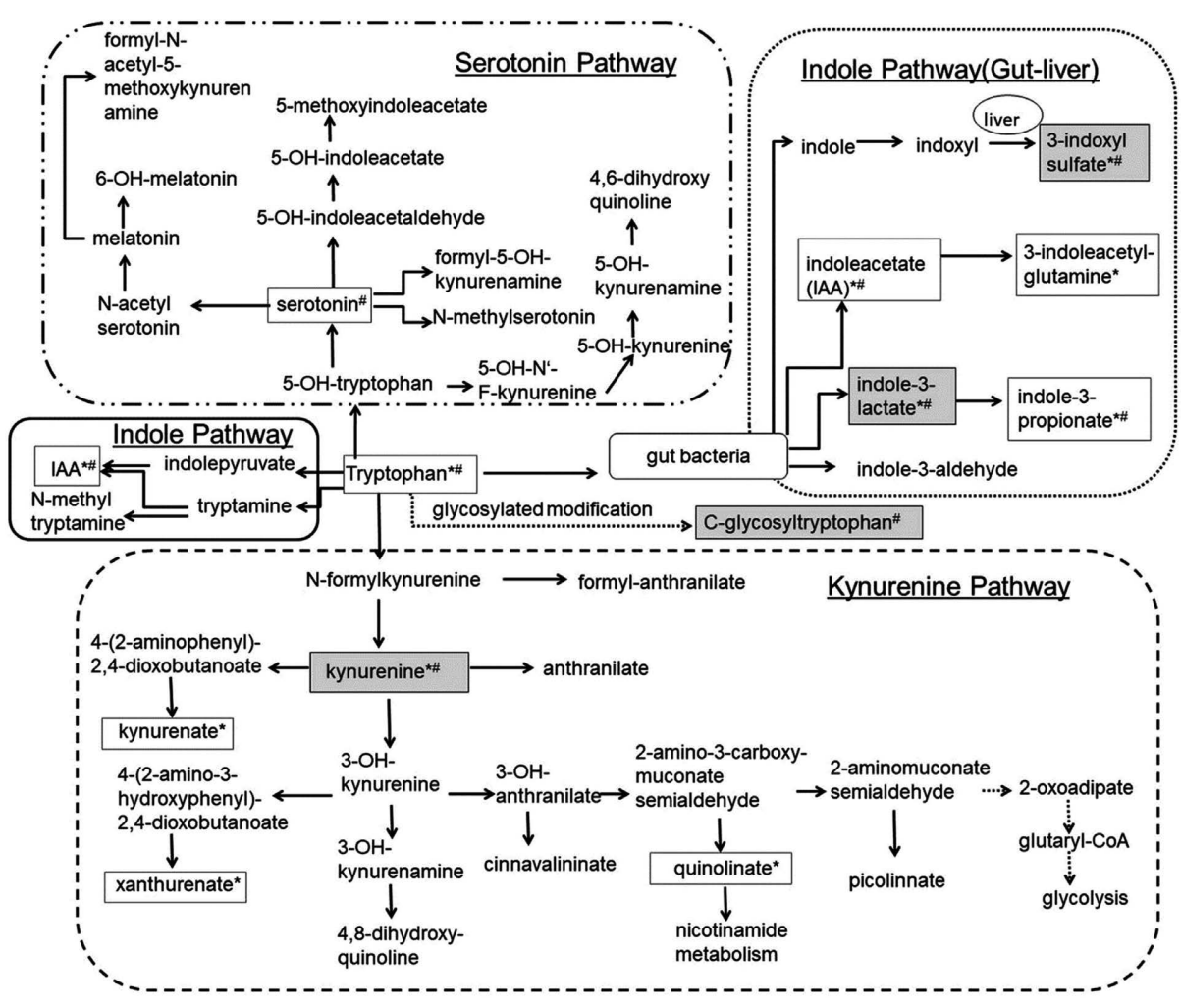

Figure 2. L-Tryptophan (L-Try) is an essential amino acid required for protein biosynthesis. It is also a biochemical precursor of metabolites that significantly affect mammalian physiology, including gastrointestinal functions, immunity, metabolism, and the nervous system. In the gastrointestinal tract L-Try metabolism can follow three significant pathways, all of which are influenced by the gut microbiota: 1) the kynurenine pathway (KP) in both immune and epithelial cells, 2) the serotonin (5-hydroxytryptamine, 5-HT; production pathway in enterochromaffin cells (ECCs), a specialized subtype of intestinal epithelial cell, and 3) direct transformation by the gutbiota of L-Try into several molecules, including ligands of the aryl hydrocarbon receptor (AhR). In mammalian cells, most L-Trp is metabolized via the KP, while the remainder is utilized in the synthesis of 5-HT and melatonin (MT). In KP, L-Trp is catabolized into the unstable derivative $\mathrm{N}$-formyl-L-kynurenine (NFK) by rate-limiting enzymes tryptophan 2,3-dioxygenase (TDO) and indoleamine 2,3-dioxygenases (IDO1/IDO2). Globally, the enzymes of KP are expressed in a tissue-specific manner. TDO is expressed in the liver, whereas IDO1 is expressed in many cell types and tissues and is inducible by cytokines NFK is rapidly metabolized by kynurenine formamidase (expressed in liver, kidney, and brain) to form L-kynurenine (L-kyn). L-kyn is a crucial metabolite with potent immunoregulatory functions through its binding to AhR. L-kyn is mainly metabolized by kynurenine monooxygenase (KMO) to form 3-hydroxykynurenine (3-HK). 3-HK is then degraded to 3-hydroxyanthranilic acid (3-HAA) by kynureninase (KYNU). KYNU is subsequently metabolized to 2-amino-3-carboxymuconic 6-semialdehyde (ACMS) by 3-hydroxyanthranilic acid 3,4-dioxygenase (3-HAO). The former is expressed in the liver, kidney, central nervous system (CNS), and placenta, while the latter has a broad tissue distribution. ACMS can be cyclized to quinolinic acid (QUIN) or metabolized by the enzyme 2-amino-3-carboxymuconate-semialdehyde decarboxylase (ACMSD), found mainly in the kidney and to a lesser extent in the liver, and responsible for the synthesis of 2-aminomuconic-6-semialdehyde (AMS). AMS is either metabolized by 2-aminomuconic semialdehyde dehydrogenase (AMSD) to result in acetyl-CoA or cyclized nonenzymatically to form picolinic acid (PICA). In the CNS, QUIN is mainly produced by microglia. It acts as a neurotoxic agent on astrocytes mainly by its selective agonist effect on ionotropic glutamate glutamatergic N-methyl-D-aspartate (NMDA) receptors. However, mechanisms determining the engagement of KP in its synthesis remain undetermined. QUIN is also a precursor for the de novo synthesis pathway for NAD via the enzyme quinolinate phosphoribosyltransferase (QPRT) expressed mainly in the liver and kidney. NAD is a cofactor for numerous enzymes involved in cellular energy metabolism, adaptive responses of cells to bioenergetic and oxidative stress, and genome stability. Its deficiency affects tissues that need high cellular energy, such as the brain, gut, and skin, causing pellagra. Finally, PICA is a neuroprotective molecule whose concentration is reduced in the serum of patients with autism, and plasma and cerebrospinal fluid (CSF) of subjects who have attempted suicide. In: Cheng, Y., Li, Y., Benkowitz, P. et al. The relationship between blood metabolites of the tryptophan pathway and kidney function: a bidirectional Mendelian randomization analysis. Sci Rep 10,12675 (2020). https://doi.org/10.1038/s41598-020-69559-x. 
one of the most critical pathways that sustain inflammation for months, contributing to generalized metabolic wastage with the emergence of neuropsychiatric symptoms and symptoms related to organ-specific inflammation, such as heart failure, renal failure, dermatological lesions.

Try generates 5-HT with a vasoconstrictor action when in homeostasis; when in inflammation, the Try starts to have its vasoconstrictor action blocked by IDO-1, which has a vasodilator action.

With improved pulmonary lesions caused by the coronavirus in acute illness, the gas exchange mechanisms return to normality; that is, oximetry will indicate adequate haemoglobin saturation. However, the patient complains of tiredness, shortness of breath, symptoms that seem to be dissociated from the improving lung or with few sequelae and a still good heart. It turns out that respiratory failure exists and appears to be due to an intracellular enzyme deficit. The lack of $\mathrm{NAD} / \mathrm{NADH}+$ and the shift from inflammation to oxidative stress underlie the pathophysiology of LCS [13].

LCS seems to be caused by the intense anaerobic mechanism of energy production since the aerobic pathway was compromised, especially in severe and critical patients. However, people with a mild, oligosymptomatic disease and even people who considered themselves not infected before in the pandemic complained of LCS signals and symptoms. There is a need for us to be aware that the virus is highly transmissible and that the way it acts in people's bodies can prevent the formation of adequate antibody titres so that, even if produced, they are not identified by the available analytical methods. Thus, we have two critical aggravating factors in the diagnostic search: negative serology does not exclude the disease, and respiratory failure is not seen in monitoring, as its essence is intracellular and not haemoglobin saturation.

Once the patient progresses to CSF with an inflammatory shift to oxidative stress, there is depletion of hormones and neurotransmitters that are important in maintaining the mechanisms of vasodilation and vasoconstriction. Metabolic stress constantly activates the adrenal to produce cortisol and stimulate the production of catecholamines. However, there is little substrate for the formation of catecholamines due to the low absorption of Phe, further accentuating the exhaustion of these molecules required by oxidative stress (Figure 1).

The pathophysiology behind the chronic symptoms in LCS may be related to the virus interfering with the RIG1-MAVS pathway to decrease type I interferon (IFN) production. SARS-CoV-2 infection of white blood cells leads to elevated glycolysis, increased mitochondrial reactive oxygen species (mtROS) production, and dysregulated mitochondrial bioenergetics. Two potent antioxidants reduced viral load and proinflammatory cytokines. Elevated levels of lactate dehydrogenase (LDH) were also reported in COVID-19, which could reflect mitochondrial dysfunction, causing inflammation is by activating the NLRP3 inflammasome in immune cells. The NLRP3 inflammasome participates in the processing and releasing inflammatory cytokines, such as IL- $1 \beta$ and IL-18.

To distinguish the patients with confirmed COVID-19 who may worsen while 
treated, we justified the following threshold values of the laboratory tests done on admission: lymphocyte count $<2.59 \times 10^{9} / \mathrm{L}$, and the upper levels for total bilirubin $11.9 \mu \mathrm{mol} / \mathrm{L}$, ALT $43 \mathrm{U} / \mathrm{L}$, AST $32 \mathrm{U} / \mathrm{L}$, D-dimer $0.7 \mathrm{mg} / \mathrm{L}$, aPTT $39.9 \mathrm{~s}$, CK 247 U/L, CRP $14.3 \mathrm{mg} / \mathrm{L}$, LDH $246 \mathrm{U} / \mathrm{L}$, troponin $0.037 \mathrm{ng} / \mathrm{mL}$, ferritin 498 $\mathrm{ng} / \mathrm{mL}$ and fibrinogen $446 \mathrm{mg} / \mathrm{dL}$.

The COVID-19 death group had significantly higher total leucocyte count ( median $=8.78, \mathrm{IQR}[5.73-13.50]$ vs. median $=6.4$, IQR $[4.7-8.5] ; \mathrm{p}=0.036)$, urea $($ median $=7.05, \mathrm{IQR}[3.90-12.93]$ vs. median $=4.5, \mathrm{IQR}[3.43 .3-5.9] ; \mathrm{p}=$ 0.029 ), and potassium (median $=4.5, \mathrm{IQR}[3.96-4.90]$ vs. median $=3.9, \mathrm{IQR}$ [3.6 - 4.3]; $\mathrm{p}=0.022$ ). In contrast, decreased levels of haemoglobin (median $=$ 11.75, IQR [10.90 - 13.20] vs. median $=13.7$, IQR [11.9 - 15.2]; $\mathrm{p}=0.017)$ were reported in the COVID-19 death group compared with the recovered group.

Lactate is considered an essential substrate for mitochondria in the muscles, heart and brain during exercise and is the primary gluconeogenetic precursor in the liver and kidneys. Median whole blood lactate levels in hospitalized patients were $\mathrm{d} 1=3.3, \mathrm{~d} 7=2.6, \mathrm{~d} 14=2.2, \mathrm{~d} 28=1.9 \mathrm{mmol}$, and in ambulatory patients, $\mathrm{d} 1=1.4, \mathrm{~d} 14=1.4, \mathrm{~d} 28=1.5 \mathrm{mmol}$. Whole blood lactate decreased significantly during the clinical course and recovery in hospitalized patients $(p=0.008)$. The blood lactate levels were significantly higher in hospitalized patients than ambulatory patients (day 1 : hospitalized versus ambulatory patients $(p=0.002)$; day 28: hospitalized versus ambulatory patients $P \leq 0.0001$ ). Elevated blood lactate is strongly associated with mortality in septic patients. Lactate seems unrelated to tissue hypoxia but is likely to reflect mitochondrial dysfunction and high adrenergic stimulation. Near-normal blood lactate, indicating preserved mitochondrial function, despite a systemic hyperinflammatory state similar to sepsis. A study Median whole blood lactate levels in hospitalized patients were $\mathrm{d} 1=3.3$, $\mathrm{d} 7=2.6, \mathrm{~d} 14=2.2, \mathrm{~d} 28=1.9 \mathrm{mmol}$, and in ambulatory patients, $\mathrm{d} 1=1.4, \mathrm{~d} 14=$ $1.4, \mathrm{~d} 28=1.5 \mathrm{mmol}$. Whole blood lactate decreased significantly during the clinical course and recovery in hospitalized patients $(p=0.008)$. Lactate levels were significantly higher in hospitalized patients than ambulatory patients (day 1: hospitalized versus ambulatory patients $(p=0.002)$; day 28: versus hospitalized ambulatory patients $P \leq 0.0001)$. These data show a long time of illness in some patients, which elevated lactate in D28 after the onset of symptoms [3].

Another study assessed data from 2860 patients. In most patients (68\%), serum lactate was lower than $2 \mathrm{mmol} / \mathrm{L}$. Elevated baseline serum lactate was associated with significantly higher ICU-and 3-month mortality (53\% vs. $43 \%$, and $71 \%$ vs. $57 \%$, respectively, $p<0.001)$. In the multivariable analysis, the maximum lactate concentration on day 1 was independently associated with ICU mortality (aOR 1.06 95\% CI $1.02-1.11 ; \mathrm{p}=0.007$ ), 30-day mortality (aOR 1.07 95\% CI $1.02-1.13 ; p=0.005$ ) and 3-month mortality (aOR 1.15 95\% CI 1.08 1.24; $p<0.001$ ) after adjustment for age, gender, SOFA score, and frailty. In 826 patients with baseline lactate $\geq 2 \mathrm{mmol} / \mathrm{L}$ sufficient data to calculate the difference between maximal levels on days 1 and 2 ( $\Delta$ serum lactate) were available. A decreasing lactate concentration over time was inversely associated with ICU 
mortality after multivariate adjustment for SOFA score, age, Clinical Frailty Scale, and gender (aOR 0.60 95\% CI $0.42-0.85 ; p=0.004$ ) [3] [4] [14].

Another study evaluated a comparison between Lactate and Renin. Area under (area under the receiver operating characteristics curve, 0.682; 95\% CI, 0.503 - 0.836; ( $p=0.05$ ), but initial lactate could not (area under the receiver operating characteristics curve, $0.615 ; 95 \% \mathrm{CI}, 0.413-0.803 ; p=0.27$ ). Peak renin (area under the receiver operating characteristics curve, 0.728 ; $95 \%$ CI, $0.547-0.888$; $(p=0.01)$ and peak lactate (area under the receiver operating characteristics curve, 0.746 ; 95\% CI, $0.584-0.876$; $p=0.01$ ) demonstrated moderate discrimination. There was no significant difference in discriminative ability between initial or peak renin and lactate concentration. At each study time point, a higher proportion of renin values exceeded the threshold of normal $(40 \mathrm{pg} / \mathrm{mL})$ in nonsurvivors than in survivors, but this association was not significant for lactate [1].

Of 221 patients with MIS-A, the median age was 21 (interquartile range [IQR], 19 - 34) years, and 154 of $219(70 \%)$ with data available were men. Sixty of 169 patients (36\%) were non-Hispanic Black individuals, and 122 of 209 (58\%) had no underlying comorbidity. One hundred two of 149 patients (68\%) noted a previous symptomatic COVID-19-like illness (median, 28 [IQR, 20 - 36] days previously). Most patients with MIS-A presented with fever (197 of 205 [96\%]), hypotension (133 of 220 [60\%]), cardiac dysfunction (114 of 210 [54\%]), shortness of breath (102 of 198 [52\%]), and/or diarrhoea (102 of 197 [52\%]). The median number of organ systems involved was5 (IQR, 4 - 6). Median hospital stay was 8 (IQR, 5 - 12) days; 115 of 201 patients (57\%) were admitted to the intensive care unit; 101 of 213 (47\%) required respiratory support, and 15 of 220 (7\%) died. Most patients (176 of 195 [90\%]) had elevated markers of coagulopathy or inflammation and a positive SARS-CoV-2 serologic finding (139 of 194 [72\%]). Ten patients with MIS-A presented with Kawasaki disease [2] [16] [20].

According to the above, there is a rarity on the subject of "chronic inflammation" or "inflammatory shock" in COVID-19 patients, as most data refer to the disease in the acute phase. However, analysing the data found, it is possible to establish that tests such as LDH and Lactate can assess the patient COVID-19 in shock, which can differentiate classic septic shock from inflammatory shock caused by COVID-19. Furthermore, myocardial damage seems to be prevalent in the chronic phase of LCS, helping in the differential diagnosis.

From March 1 through March 10, 2020, the incidence of MIS-C in New York State among people younger than 21 years was 2 per 100,000 individuals vs 322 per 100,000 individuals with laboratory-confirmed SARS-CoV-2 infection. Third, there may be a diversity of phenotypes of MIS not only between individual cases but across age groups. In MIS-C, mucocutaneous manifestations were more common in the younger cohort, while in older children, myocarditis and gastrointestinal symptoms were more frequently reported. 4 In fact, in the adult cases of MIS identified by Davogustto et al., 612 of 15 patients with MIS-A (80.0\%) had gastrointestinal complications. As we learn more about MIS-A, we 
are likely to uncover additional aspects of its spectrum. Lastly, evidence for an optimal treatment strategy for MIS-A is lacking, and for now, therapy may depend on supportive medical management. In children, MIS-C therapy frequently includes intravenous immunoglobulins and glucocorticoids, which are extrapolated from the management strategies of Kawasaki disease. Further research will be needed to decide whether these same treatments can be applied to adult cases. However, initially, the mitochondrial component appears to be preserved in the acute phase of the disease, reflecting low serum lactate titers. The chronic phase appears to reflect the spoliation of enzymes necessary for the metabolic pathway of aerobic cellular respiration to be carried out correctly. Thus, hypoxemia resulting from deficient intracellular respiration despite haematosis preserved by the structural improvement of the lungs, whose acute injury by SARS-CoV-2 triggered respiratory failure. Thus, the patient may present in chronic disease with adequate $\mathrm{O}_{2}$ saturation, with signs and symptoms of respiratory failure in progress, especially if the patient presents acute deterioration evolving to inflammatory shock [1] [21].

However, recent studies are beginning to challenge the centrality of cytokine storms in severe COVID-19 cases. For instance, a study demonstrated that inflammatory cytokine elevations in patients with severe and critical COVID-19, including elevations of IL-6, are profoundly lower than those reported in patients with comparable conditions such as acute respiratory distress syndrome (ARDS), which is unrelated to COVID-19 and sepsis. In the same study, several non-cytokine biomarkers, including D-dimer, C-reactive protein, and ferritin, were elevated to a similar or greater extent in COVID-19 patients than in patients with sepsis or ARDS. No statistically significant differences were observed in baseline levels of multiple cytokines between COVID-19 and sepsis patients. The COVID-19 complications are now mainly associated with immunosuppression, especially lymphopenia rather than cytokine storm. Increased T lymphopenia, particularlyCD4+ and CD8+ T cells, have been shown to decrease markedly in COVID-19 patients, implying damage of lymphocytes by the SARS-CoV-2 virus. Moreover, unlike episodic cytokine storm, lymphopenia is continuous in critically ill COVID-19 patients and leads to increased secondary infections.

\subsection{Changes in the Glycolytic Pathway and Adenosine Production}

Many viruses alter host cell metabolism similarly to the Warburg effect, increasing glycolysis and producing rapid energy and substrates for nucleotide replication and specific protein synthesis. Increased aerobic glycolysis has been demonstrated in Zika virus (ZIKV) with up-regulation of glycolytic genes, including membrane glucose transporter 1 (GLUT1), various glycolysis enzymes, and MCT4, the transporter that expels lactate out of cells. In intestinal cells, the coronavirus transmissible gastroenteritis virus (TGEV) increases glucose uptake via the apical transporters of Nap-dependent glucose transporter 1 (SGLT1) and basal glucose transporter 2 (GLUT2), and uptake stimulated by Epidermal 
growth factor receptor (EGFR) Host cell metabolism and activation of signalling pathways are reprogrammed by viral proteins, as shown in adenovirus-E4-ORF1. The two main signalling pathways-PI3/AKT/mTOR and MAPK/ERK - promote replication of the Middle East Respiratory Syndrome coronavirus (MERS-CoV), enterovirus 71 (EV71) and ZIKA. In MERS-CoV, $\mathrm{PI} 3 / \mathrm{AKT} / \mathrm{mTOR}$ and MAPK/ERK inhibitors (including rapamycin) inhibit virus replication in vitro, regardless of whether the inhibitors are introduced before or after viral infection. Thus, considering that SARS-CoV-2 and MERS-CoV are beta-coronaviruses that share numerous similarities, we hypothesize that SARS-CoV-2 replication is supported in airway cells by the Warburg effect promoted by PI3 pathways./AKT/mTOR and MAPK/ERK. Studies need to prioritize the following considerations:

That metabolism may be cell type dependent.

The Warburg effect can be activated independently of the infection itself (e.g., in response to hypoxia).

Moreover, the virus could promote alternative pathways (glutaminolysis) to adapt nutrient conditions to biosynthetic or bioenergetic demands.

The energy consumption of metabolic activities in normal cells depends mainly on mitochondrial oxidative phosphorylation (OXPHOS), which is efficient and generates more adenosine triphosphate (ATP) than glycolysis. COVID-19 causes severe hypoxia due to lung damage and interference with tryptophan metabolism. Hypoxemia generates large production of adenosine, but not only in acute infection.

Although the lungs can recover, or the patient can adapt to the sequelae caused in the acute phase of the disease, dysmetabolism generated with the possibility of chronic inflammation due to the internalization of ACE-2 and interference in the tryptophan cycle, changing the NAD/NADH+. Therefore, it promotes the deflection of aerobic respiration to beta-oxidation of fatty acids and creates an environment for oxidative stress and its consequences, such as insulin resistance and atheroma.

Two primary nutrients captured and utilized by cancer cells are glucose and glutamine. These two nutrients contribute to most cellular carbon sources used for biogenesis. Glucose catabolism generates ATP, NADPH and other biomasses for reductant biosynthesis and ROS detoxification. In association with the TCA cycle, glutamine metabolism provides not only a carbon source but also NADH, $\mathrm{NH} 3+$ and other essential intermediates for lipid biosynthesis, amino acid synthesis, and cellular acid detoxification. Monocytes enter sites of microbial or sterile inflammation as the first line of defense of the immune system and initiate pro-inflammatory effector mechanisms. We show that activation with bacterial lipopolysaccharide (LPS) induces them to undergo a metabolic shift toward aerobic glycolysis, similar to the Warburg effect observed in cancer cells. Monocytes, macrophages and dendritic cells [22] [23] undergo a switch from oxidative phosphorylation to glycolysis in response to toll-like receptor 4 activation by LPS, the so-called Warburg effect [24] [25]. 
Glycolysis supplies metabolic intermediates for other biosynthetic pathways necessary for cellular growth and differentiation during the glycolytic switch. In addition to glycolysis, the pentose phosphate pathway (PPP), the hexosamine pathway and the glutaminolysis are increased upon activation. There is a compensatory increase in anaerobic glycolysis to maintain the cell energy sources. Consequently, intracellular glycogen stores are rapidly depleted, and lactic acid accumulates, leading to decreased activity of many cellular enzymes (due to decreased $\mathrm{pH}$ levels). In addition, new evidence revealed that the upregulation of glycolysis is not just for ATP synthesis but also for the synthesis of biomass such as ribonucleotides and amino acids as well as reduced nicotinamide adenine dinucleotide phosphate (NADPH) production, which can remove reactive oxygen species (ROS) generated by cancer cells' accelerated metabolism under hypoxic conditions.

Metabolic syndrome, diabetes, obesity, hypertension, cardiovascular disorders and chronic nephropathy are frequently observed in patients with COVID-19. These conditions promote the occurrence of a low-grade inflammatory state, a condition that increases with ageing. The chronic state of inflammation impairs endothelial function, immune response and antioxidant defence while increasing the development of cancers. Ageing increases the Warburg effect on cells via oxidative stress and the production of reactive oxygen species (ROS), promoting the activation of HIF-1a; Mitochondrial senescence, decreased oxidative phosphorylation (OXPHOS), dysfunction of the AMPK and p53 pathways conspire to promote aerobic glycolysis in elderly patients.

Glycolysis orchestrates the behaviour of inflammatory cells. Upon inflammation, immune cells are reprogrammed towards glycolysis, enhancing this pathway at the expense of OXPHOS and, in macrophages, imbalance [24] [26] in the TCA cycle. Consequently, glycolysis and broken TCA cycle-released metabolites such as lactate, citrate or succinate accumulate in immune cells, promoting signalling, post-translational and epigenetic modifications that drive the inflammatory response. Moreover, glycolytic intermediates fuel other pathways such as the PPP, which provides biosynthetic molecules, and the hexosamine one generates UDP-GlcNAc for glycation reactions.

The pentose phosphate pathway (PPP). The PPP branches after the first step of glycolysis and goes back to fructose 6-phosphate and glyceraldehyde 3-phosphate in the glycolytic and gluconeogenic pathway. The PPP produces R5P and NADPH for biosynthesis and redox regulation. NADPH from the PPP converts oxidized glutathione (GSSG) to reduced glutathione (GSH). GSH elicits insulin granule exocytosis via SENP1. However, NADPH might inhibit insulin secretion by promoting NADPH oxidases (NOXs). Hyperglycemia decreases G6PD activity by activating protein kinase A (PKA) and increasing intracellular oxidative stress, leading to chronic kidney injury and diabetic kidney disease (DKD).

$\beta$-oxidation is the catabolic process by which fatty acid molecules are broken down to generate acetyl-CoA and NADH and FADH2, which are used in the 
electron transport chain to produce ATP. However, $\beta$-oxidation occurs at two sites, the peroxisome in the cytosol and the mitochondrion using long-chain fatty acids and medium-chain fatty acids, respectively.

Carnitine shuttle is a rate-limiting step of fatty acid oxidation in mitochondria, and therefore fatty acid oxidation can be regulated in this step [23] [24] [25] [26] [27].

Malonyl CoA, a fatty acid synthesis intermediate, presents in the cytosol, is an inhibitor of carnitine acyltransferase I. This indicates that when fatty acid synthesis is in progress, fatty acid oxidation cannot coincide as the carnitine shuttle is impaired by inhibition of carnitine acyltransferase I.

Oxidation of fatty acids is also regulated by the ratio of acetyl CoA to CoA: as the ratio increases, the thiolase reaction that requires $\operatorname{CoA}$ (the enzyme that participates in $\beta$-oxidation) decreases.

When the $[\mathrm{NADH}] /[\mathrm{NAD}+]$ ratio increases, the $\beta$-hydroxy acyl-CoA dehydrogenase enzyme is inhibited.

The Warburg effect is the regeneration of NAD + NADH in the pyruvate-to-lactate step that completes the aerobic glycolysis. In this scenario, NADH produced by glyceraldehyde phosphate dehydrogenase (GAPDH) must be consumed to regenerate NAD+ and keep glycolysis active. This high rate of glycolysis allows supply lines to remain open, such as siphon 3-phosphoglycerate (3PG) to serine for one-carbon metabolism mediated production of NADPH nucleotides. A possible hypothesis is that by significantly decreasing NAD+ (both by consumption and deficit of tryptophane), the Warburg effect is activated to increase the NAD/NADH+ ratio.

One of the possibilities that $\mathrm{LDH}$ is a good reference for diagnosing inflammation in COVID-19, both in the acute phase and in LCS, is to consider that, due to the Warburg Effect and the intensification of the glycolytic pathway, there is a greater need for LDH expression. However, due to the Tryptophane deficit, there is little substrate to guarantee the formation of NAD, generating a deficiency in the NAD/NADH+ balance for adequate aerobic cell respiration [22] [23] [24].

LDH ((S)-lactate: NAD+ oxidoreductase; EC 1.1.1.27) is a family of NAD+dependent enzymes that catalyse the inter-conversion between pyruvate and lactate, with concomitant oxidation/reduction of the cofactor (NAD+/NADH). $\mathrm{LDH}$ simultaneously regenerates NAD+ molecules is certainly the most important step required for glycolysis to proceed and release cellular energy. LDHA is located mainly in the cytoplasm, but it has also been found to bind single-stranded DNA in the nucleus, although its involvement in DNA metabolism is not well understood. LDHA has been acknowledged as a valuable predictive and prognostic marker; its overexpression is associated with cancer invasiveness; elevated serum lactate levels correlate with poor prognosis and resistance to chemo- and radiotherapy LDHA expression is regulated by c-My and HIF-1. Moreover, tyrosine phosphorylation by the oncogenic FGFR1 receptor was found to activate LDHA, thereby promoting the Warburg effect in cancer cells. 
BCAAs and fatty acids can participate in the TCA cycle and glycolysis. The catabolism of BCAAs and fatty acids can interplay through these metabolites and ultimately impact mitochondrial ATP production. Enhanced fatty acid oxidation increased the ratio of mitochondrial acetyl-CoA/CoA and NADH/NAD+, leading to the inactivation of pyruvate dehydrogenase. Increased the intracellular level of citrate, thereby inhibiting the accumulation of phosphofructokinase and glucose-6-phosphate, which hindered the normal progress of glycolysis.

Under normal oxygenated conditions, the rate of ATP catabolism and anabolism are equal, and the concentration of ATP is maintained at a much higher level (at least 10 times higher) than that of ADP. Under conditions of tissue stress or trauma, such as ischemia or hypoxia, however, the oxygen supply is inadequate for oxidative phosphorylation of ADP to regenerate ATP, and the ATP/ADP ratio is compromised. The increasing concentration of ADP results in an increase in the concentration of AMP, which in turn is hydrolyzed to adenosine. The most important source of adenosine quantitatively is the AMP-selective cytosolic 5'-nucleotidase (c-NI), which has been suggested to be responsible for approximately $76 \%$ of adenosine production in mammalian cells. Another essential enzyme responsible for the production of adenosine via hydrolysis of AMP is ecto-5'-nucleotidase (e-N), a glycoprotein found primarily in the plasma membrane of eukaryotic cells. In addition to these two enzymes, adenosine can also be produced from S-adenosylhomocysteine (SAH) via SAH-hydrolase [28] [29] [30].

Adenosine is a vital signalling nucleoside that orchestrates cell and tissue adaptation under energy depletion and ischemic/hypoxic conditions by activating four G protein-coupled receptors (GPCR). The regulation and generation of extracellular adenosine in response to stress is critical in tissue protection. Studies in rats and humans have reported that extracellular adenosine signalling plays a beneficial role during acute states.

Adenosine is ubiquitously produced in almost every cell in our body under physiological conditions and subsequently produced under hypoxia or energy depletion conditions. As a critical building block and intermediate metabolite of nucleic acids, adenosine is a key signalling molecule that orchestrates the cellular response to hypoxia, energy depletion and tissue damage, activating its $G$ protein-coupled receptors (GPCR) in various types of cells. Under normal physiological conditions, adenosine extracellular and intracellular levels are in the nanomolar range. However, under stressful conditions, ATP is released from damaged cells, such as endothelial cells, neutrophils, and glial cells, through transmembrane protein channels, including pannexins or connexins, and subsequently dephosphorylated to extracellular adenosine by ectonucleotidases including CD39, which converts ATP to ADP/AMP and CD73 which converts AMP to adenosine.

Adenosine is well known to be induced under hypoxic conditions, and patients with FD are in a chronic state of hypoxia. Elevated plasma adenosine through activation of the ADORA2B receptor in the erythrocyte leads to sick- 
ling, haemolysis, and damage to various erythrocyte tissues in transgenic mice with sickle cells (Figure 3) [28] [29] [30] [31].

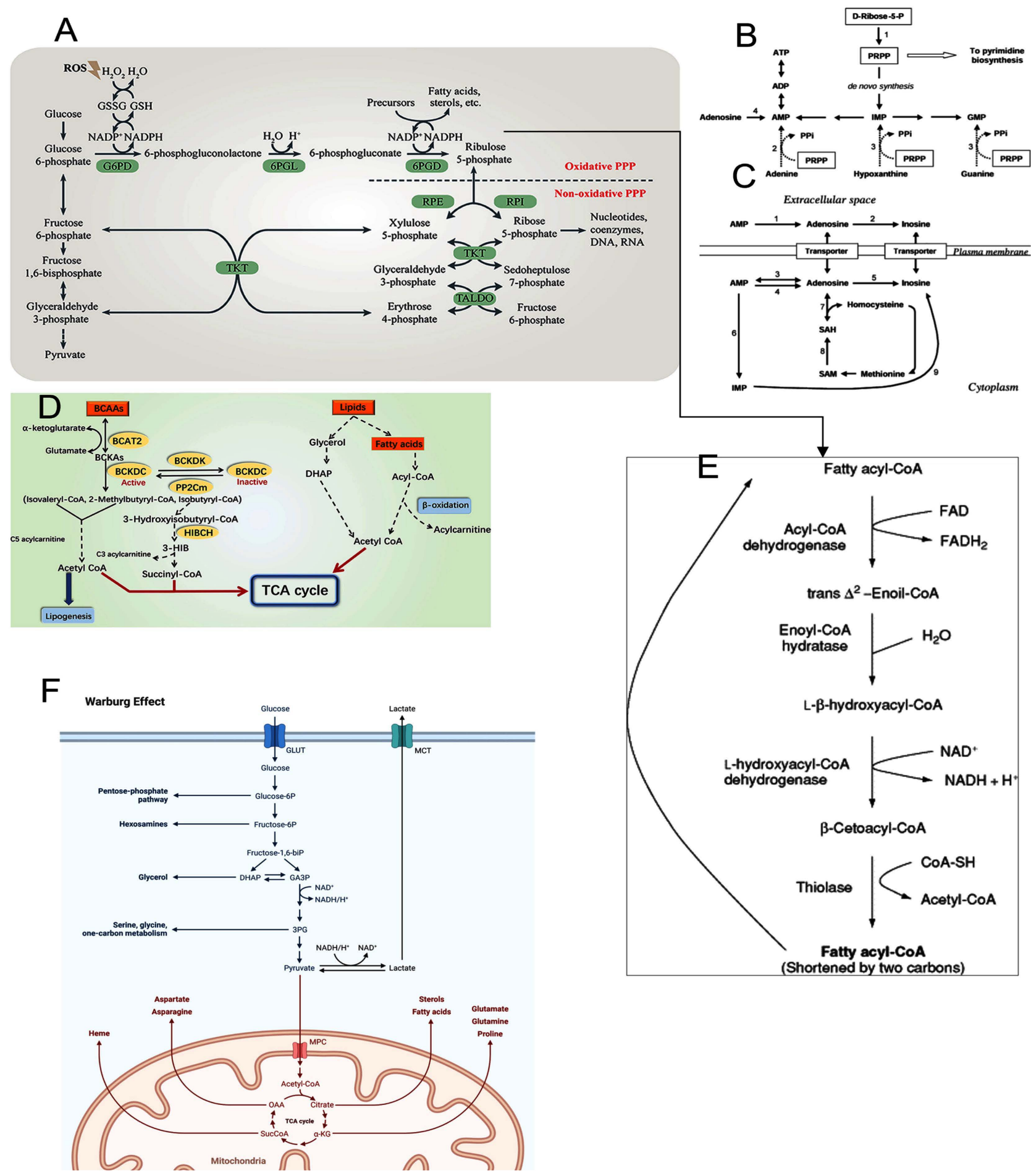

Figure 3. (A) Activation of the pentose pathway to obtain ATP. (B)-(C) Adenosine production. (D) Hyperglycemia appears due to oxidative stress and BCAA metabolism. (E) The beta-oxidation of fatty acids can form ATP, however, in COVID-19, the citric acid cycle is truncated, and there is a deficit of NAD+ as a hydrogen acceptor. (F) The innate immunity cells and the tissue cells due to hypoxia in the acute phase of the disease obtain energy through the Warburg effect with optimization of the glycolytic pathway in the presence of a medium rich in glucose and pyruvate formation, consequently, an increase in LDH expression. 


\subsection{Organizing Pneumonia and COVID-19 Chronic Sequelae}

Organizing pneumonia appears as a complication of the late phase of COVID-19. Corticosteroids are standard therapy for organizing pneumonia, but the question of whether a high-dose corticosteroid approach would be beneficial for patients with organizing pneumonia secondary to COVID-19 remains unanswered.

Patients undergoing chest computed tomography due to maintenance of hypoxemia, which presented a pattern compatible with organizing pneumonia. $\mathrm{Pa}-$ tients were treated with high-dose corticosteroids $(1 \mathrm{mg} / \mathrm{kg}$ VO prednisone), with marked clinical improvement and decreased oxygen flow rate demand. They were discharged after an average length of stay of 6.3 days.

OP or COVID-19 sequelae such as laminar atelectasis, consolidations, groundglass regions, previous COVID-19 enhanced pleural effusions and may contribute to the search for a plausible diagnostic hypothesis [32] [33] [34] [35] [36].

OP usually appears in hypotheses with the activation of inflammation via IL-6, so it can appear in any situation where there is a cytokine storm with this cytokine pattern. That is, OP is not pathognomonic of COVID-19 [32] [33] [34] [35] [36] (Figure 4).

\section{Discussion}

The review used for this article shows that CLS is more easily recognized in paediatric patients than in adult patients and this fact remains a great mystery to be deciphered for the author of this text. LCS (MISc) is treated with corticosteroids and immunoglobulin, while LCS (MIS-A) is still neglected as a nosological entity and is not treated.

In the author's view, LCS is an inflammatory entity caused by SARS-CoV-2 infection due to the internalization of ACE-2 and mainly by the shift from the aerobic axis of cellular respiration to the oxidative stress axis due to the enzyme and intracellular antioxidant deficit, intensifying insulin resistance, initial hypercortisolism and shifting the immune response to innate immunity, which signals the rarity of the disease, as stated in an article already submitted for publication. Furthermore, bradycardia in the presence of fever or hyperthermia may result from cell damage with subsequent production of adenosine, justifying the pathophysiology of the Faget's signal (Figure 1).

It is essential to consider that in ISC, due to its chronic status, the alveoli may already be recovered, or there was an adaptation to the sequelae condition after COVID-19; for this reason, haemoglobin saturation may be normal on pulse oximetry or arterial blood gases. The defect that leads the patient to respiratory failure is intracellular and makes the diagnosis more complicated but not impossible since LDH, elevated triglycerides and cholesterol may indicate that the inflammatory axis is on oxidative stress due to the enzymatic failure necessary for adequate aerobic respiration. 

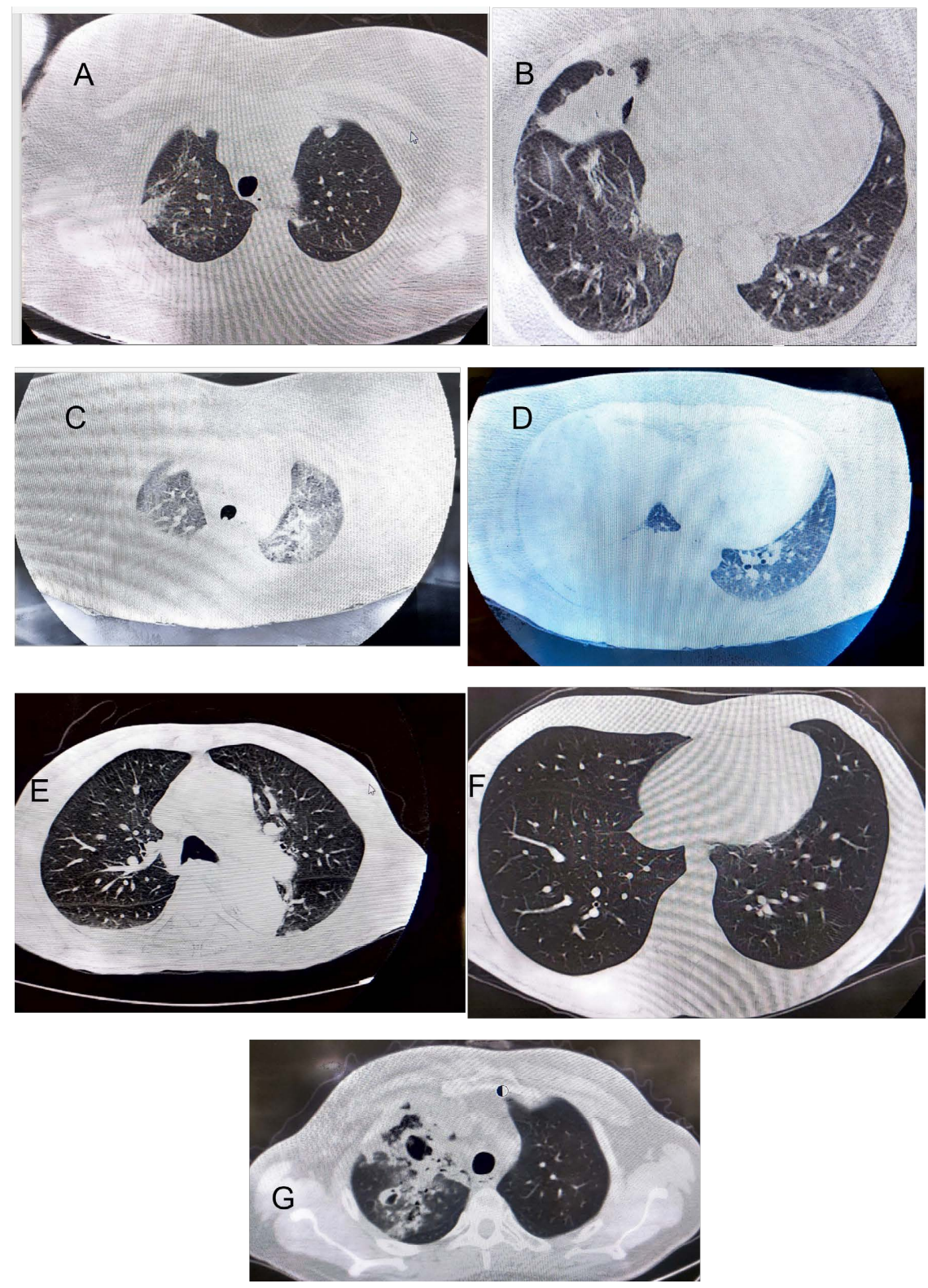

Figure 4. (A) 80-year-old woman; (B) 67-year-old man; (C) (D) 28-year-old woman; (E) (F) 66-year-old man; (G) 53-year-old man. All of them with systemic inflammation post COVID-19. Computed tomography images show patients hospitalized in the same period in Hospital do Server Estadual ward. These patients have extensive investigation for tuberculosis and fungi. Negatives. Epidemiology, at a time of SARS-CoV-2 pandemic, the images are not coincidences but are part of the progression to organizing pneumonia, which is not pathognomonic of COVID-19, but is very prevalent when the disease progresses to inflammatory syndrome multisystem. Otherwise, the most common sequelae are laminar atelectasis, mosaic infiltrates and consolidations that resemble calcified nodules.

LCS has a chronic inflammatory status that leads the post-COVID-19 patient to physical and metabolic exhaustion, leading the patient to vasoplegic shock due to exhaustion of catecholamines, since with the internalization of ACE-2, there is less intestinal absorption of amino acids essential as Phenylalanine (Phe) and Tryptophan (Try), with serotonergic involvement as well. 
The Kynurenine pathway is initiated by converting L-tryptophan (Try) by either the enzymes tryptophan-2,3-dioxygenase or indoleamine 2,3-dioxygenase forming formyl-kynurenine, which is then further degraded to kynurenine, the precursor of several bioactive compounds, including kynurenic acid, quinolinic acid, picolinic acid, and 3-hydroxyanthranilic acid. The pathway is responsible for over $90 \%$ of tryptophan metabolism in the periphery.

Kynureninase is a pyridoxal phosphate-dependent enzyme inhibited by oestrogen and metabolites, with vitamin B6 deficiency and inhibition. There is an increase in Kyn, HK, XA, and Hal and a decrease in KA and AA because of the available vitamin B-6 deficiency simulation in which the activities of the enzymes were not wholly reduced [12] [13].

Naive CD4+T cells can differentiate into T helper (Th) cells, Th1, Th2, and Th17 lineages and regulatory $\mathrm{T}$ (Treg or $\mathrm{CD} 3+\mathrm{CD} 4+\mathrm{FOXP} 3+)$ cells. -6 and transforming growth factor $\beta$ (TGF $\beta$ ) is considered critical for the differentiation into Th17 cells. IL-21 and IL-23 pathways are involved in IL-6-programmed Th-17 cell differentiation. Various cytokines regulate Th17 differentiation. TGF- $\beta$ and IL- 6 induced Th17 differentiation in mice, and IL- $1 \beta$ but not TGF- $\beta$ has been shown to develop Th17 cells together with IL- 6 in humans.

The development of Th17 cells is negatively regulated by IFN- $\gamma$, IL-27, and IL-2, the signals of which are dependent on Stat1 (IFN- $\gamma$ and IL-27) and Stat5 (IL-2), respectively. The orphan nuclear receptors, retinoid-related orphan receptor $\gamma(\operatorname{ROR} \gamma)$ and ROR $\alpha$ have been identified as the key transcription factors that determine the differentiation of Th17 lineage. AhR is involved in the differentiation of Th17 cells by regulating Stat 1 activation, which suppresses Th17 cell differentiation under Th17-polarizing conditions (Figure 2).

Cellular damage from hypoxemia produces too much adenosine (ADA) in response to injury. ADA performs negative feedback for multiple pathways of intracellular aerobiosis and stimulates bradycardia when in excess, which can cause cardiorespiratory arrest. Adenosine limits the degree of vascular injury during ischemia and reperfusion by inhibition of oxygen radical release from activated neutrophils, thereby preventing endothelial cell damage, and by inhibition of platelet aggregation. These effects help to preserve endothelial cell function and microvascular perfusion. Long-term exposure to adenosine may also induce coronary angiogenesis [37]-[42].

Due to the need for ATP, the pentose pathway is linked to compensate for the energy deficit through oxidative phosphorylation. However, the citric acid pathway is enzymatically blocked, leaving the cell to carry out the Warburg effect, facilitating oncogenes and protooncogenes to be activated, orchestrating a status of tolerance and immunosuppression. With the activation of the Warburg effect, innate immune cells can produce ATP in a medium nourished by Glucose, while in hypoglycaemic or normoglycemic status, the oxidative phosphorylation pathway is preferred. For this reason, it is necessary to maintain glycaemic control in severe COVID-19 patients, tending to form ATP through a non-injurious and tumorigenic pathway. Elevated LDH shows intense glycolytic 
activity, thus the need to provide substrates to maintain the citric acid pathway and oxidative phosphorylation patent.

Regarding sepsis and Septic Shock, we have an acute event that, if left untreated, leads to death. Aerobic breathing pathways are not impeded, but tissue perfusion may be impaired, causing an increase in lactate with low LDH. In ISC, however, the DHL is very high due to tissue damage and blockage of the aerobic intracellular respiration pathway (Figure 3).

\section{Conclusion}

Post-COVID-19 Inflammatory Syndrome is well recognized in children, and we must recognize that it is part of the pathophysiology of the disease in adults as well. Ignoring the differential diagnosis between classic septic shock and inflammatory vasoplegic shock is a life-or-death condition for the patient. We must carry out trials identifying features that facilitate the diagnosis. Based on the survey carried out in this article, I consider LDH, Lactate, C-Reactive Protein, bradycardia (in situations in which the body demands compensatory tachycardia) to be essential tests to make this difference. Multisystem Inflammatory Syndrome in adults or what this article calls ISC (Inflammatory Shock after COVID-19) should be treated with high doses of corticosteroids or immunoglobulins. ISC is the same or similar entity as the MISc, an inflammatory status that has been seen in paediatric patients. LDH shows a magnitude of the Warburg effect and may indicate that COVID-19 patients are in LCS and chronic inflammation. LDH shows a magnitude of Warburg effect and may indicate that patients with COVID-19 are in LCS and chronic inflammation. Normalizing blood glucose is a way of forcing ATP production by oxidizing phosphorylation.

\section{Acknowledgements}

To the Public Servant Hospital of the State of São Paulo (HSPE).

To the HSPE Emergency Department.

To the Emergency Department of the University Hospital of the Faculty of Medicine of University of São Paulo (USP)—Main Campus_São Paulo.

To all patients that suffer from COVID-19 and its sequelae.

\section{Elaboration of the Article}

The article was entirely written by the author.

\section{Ethical Aspects}

The article does not require a consent form according to the National Research Ethics Committee of Brazil (Conep). All ethical aspects are in accordance with Conep standards.

\section{Conflicts of Interest}

The author declares no conflicts of interest regarding the publication of this paper. 


\section{References}

[1] Patel, P., Decuir, J., Abrams, J., Campbell, A.P., Godfred-Cato, S. and Belay, E.D. (2021) Clinical Characteristics of Multisystem Inflammatory Syndrome in Adults a Systematic Review. JAMA Network Open, 4, Article ID: 2126456. https://doi.org/10.1001/jamanetworkopen.2021.26456

[2] Chow, E.J. (2021) The Multisystem Inflammatory Syndrome in Adults with SARS-CoV-2 Infection-Another Piece of an Expanding Puzzle. JAMA Network Open, 4, Article ID: e2110344.

https://doi.org/10.1001/jamanetworkopen.2021.10344

[3] Wang, Y., Yao, R., Zhang, L., Xie, X., Chen, R. and Ren, Z. (2019) IDO and intra-Tumoral Neutrophils Were Independent Prognostic Factors for Overall Survival for Hepatocellular Carcinoma. Journal of Clinical Laboratory Analysis, 33, Article ID: e22872. https://doi.org/10.1002/jcla.22872

[4] Gasperi, V., Sibilano, M., Savini, I. and Catani, M.V. (2019) Niacin in the Central Nervous System: An Update of Biological Aspects and Clinical Applications. International Journal of Molecular Sciences, 20, Article No. 974. https://doi.org/10.3390/ijms20040974

[5] Westbrook, R., Chung, T., Lovett, J., Ward, C., Joca, H., Yang, H., et al. (2020) Kynurenines Link Chronic Inflammation to Functional Decline and Physical Frailty. JCI Insight, 5, Article ID: e136091. https://doi.org/10.1172/jci.insight.136091

[6] Favennec, M., Hennart, B., Caiazzo, R., Leloire, A., Yengo, L., Verbanck, M., et al. (2015) The Kynurenine Pathway Is Activated in Human Obesity and Shifted toward Kynurenine Monooxygenase Activation. Obesity, 23, 2066-2074.

https://doi.org/10.1002/oby.21199

[7] Chen, L.-M., Bao, C.H., Wu, Y., Liang, S.H., Wang, D., Wu, L.Y., et al. (2021) Tryptophan-Kynurenine Metabolism: A Link between the Gut and Brain for Depression in Inflammatory Bowel Disease. Journal of Neuroinflammation, 18, Article No. 135. https://doi.org/10.1186/s12974-021-02175-2

[8] Sakurai, M., Yamamoto, Y., Kanayama, N., Hasegawa, M., Mouri, A., Takemura, M., et al. (2020) Serum Metabolic Profiles of the Tryptophan-Kynurenine Pathway in the High Risk Subjects of Major Depressive Disorder. Scientific Reports, 10, Article No. 1961. https://doi.org/10.1038/s41598-020-58806-w

[9] Vakhshoori, M., Heidarpour, M., Bondariyan, N., Sadeghpour, N. and Mousavi, Z. (2021) Adrenal Insufficiency in Coronavirus Disease 2019 (COVID-19)-Infected Patients without Preexisting Adrenal Diseases: A Systematic Literature Review. International Journal of Endocrinology, 2021, Article ID: 2271514.

https://doi.org/10.1155/2021/2271514

[10] Talaei, F., Bouma, H.R., van der Graaf, A.C., Strijkstra, A.M., Schmidt, M. and Henning, R.H. (2011) Serotonin and Dopamine Protect from Hypothermia/Rewarming Damage through the CBS/ H2S Pathway. PLoS ONE, 6, Article ID: e22568. https://doi.org/10.1371/journal.pone.0022568

[11] Zhou, H., Peng, X., Hu, J., Wang, L., Luo, H., Zhang, J., et al. (2021) DsbA-L Deficiency in $\mathrm{T}$ Cells Promotes Diet-Induced Thermogenesis through Suppressing IFN- $\gamma$ Production. Nature Communications, 12, Article No. 326. https://doi.org/10.1038/s41467-020-20665-4

[12] Thomas, T., Stefanoni, D., Reisz, J.A., Nemkov, T., Bertolone, L., Francis, R.O., et al. (2020) COVID-19 Infection Alters Kynurenine and Fatty Acid Metabolism, Correlating with IL-6 Levels and Renal Status. JCI Insight, 5, Article ID: e140327. 
https://doi.org/10.1172/jci.insight.140327

[13] Page-Wilson, G., Arakawa, R., Nemeth, S., Bell, F., Girvin, Z., Tuohy, M.-C., et al. (2021) Obesity Is Independently Associated with Septic Shock, Renal Complications, and Mortality in a Multiracial Patient Cohort Hospitalized with COVID-19. PLoS ONE, 16, Article ID: e0255811. https://doi.org/10.1371/journal.pone.0255811

[14] Edalatifard, M., Akhtari, M., Salehi, M., Naderi, Z., Jamshidi, A., Mostafaei, S., et al. (2020) Intravenous Methylprednisolone Pulse as a Treatment for Hospitalised Severe Covid-19 Patients: Results from a Randomised Controlled Clinical Trial. European Respiratory Journal, 56, Article ID: 2002808.

https://doi.org/10.1183/13993003.02808-2020

[15] de Assis Barros D’Elia Zanella, L., de Sá Paraskevopoulos, D., de Lima Galvão, L. and Yamaguti, A. (2021) Methylprednisolone Pulse Therapy in COVID-19 as the First Choice for Public Health: When Right Timing Breaks Controversies-Emergency Guide. Open Journal of Emergency Medicine, 9, 84-114. https://doi.org/10.4236/ojem.2021.93010

[16] Bruno, R.R., Wernly, B., Flaatten, H., Fjølner, J., Artigas, A., Bollen Pinto, B., et al. (2021) Lactate Is Associated with Mortality in Very Old Intensive Care Patients Suffering from COVID-19: Results from an International Observational Study of 2860 Patients. Annals of Intensive Care, 11.

[17] Chau, V.Q., Giustino, G., Mahmood, K., Oliveros, E., Neibart, E., Oloomi, M., et al. (2020) Cardiogenic Shock and Hyperinflammatory Syndrome in Young Males with COVID-19. Circulation: Heart Failure, 13, 556-559. https://doi.org/10.1161/CIRCHEARTFAILURE.120.007485

[18] Arina, P., Moro, V., Baso, B., Baxter-Derrington, C. and Singer, M. (2021) Sepsis in Severe COVID-19 Is Rarely Septic Shock: A Retrospective Single-Centre Cohort Study. British Journal of Anaesthesia, 127, e182-e185.

https://doi.org/10.1016/j.bja.2021.08.007

[19] Poston, J.T., Patel, B.K. and Davis, A.M. (2020) Management of Critically Ill Adults with COVID-19. JAMA, 323, 1839-1841. https://doi.org/10.1001/jama.2020.4914

[20] Iepsen, U.W., Plovsing, R.R., Tjelle, K., Foss, N.B., Meyhoff, C.S., Ryrsø, C.K., et al. (2021) The Role of Lactate in Sepsis and COVID-19: Perspective from Contracting Skeletal Muscle Metabolism. Experimental Physiology.

https://doi.org/10.1113/EP089474

[21] Ibrahim, M.E., AL-Aklobi, O.S., Abomughaid, M.M. and Al-Ghamdi, M.A. (2021) Epidemiological, Clinical, and Laboratory Findings for Patients of Different Age Groups with Confirmed Coronavirus Disease 2019 (COVID-19) in a Hospital in Saudi Arabia. PLoS ONE, 16, Article ID: e0250955.

https://doi.org/10.1371/journal.pone.0250955

[22] Nagy, C. and Haschemi, A. (2015) Time and Demand Are Two Critical Dimensions of Immunometabolism: The Process of Macrophage Activation and the Pentose Phosphate Pathway. Frontiers in Immunology, 6, Article No. 164. https://doi.org/10.3389/fimmu.2015.00164

[23] Chen, X., Qian, Y. and Wu, S. (2015) The Warburg Effect: Evolving Interpretations of An Established Concept. Free Radical Biology and Medicine, 79, 253-263. https://doi.org/10.1016/j.freeradbiomed.2014.08.027

[24] Jiang, P., Du, W. and Wu, M. (2014) Regulation of the Pentose Phosphate Pathway in Cancer. Protein \& Cell, 5, 592-602. https://doi.org/10.1007/s13238-014-0082-8

[25] Soto-Heredero, G., Gómez de Las Heras, M.M., Gabandé-Rodríguez, E., Oller, J. and Mittelbrunn, M. (2020) Glycolysis-A Key Player in the Inflammatory Re- 
sponse. The FEBS Journal, 287, 3350-3369. https://doi.org/10.1111/febs.15327

[26] Ye, Z., Wang, S., Zhang, C. and Zhao, Y. (2020) Coordinated Modulation of Energy Metabolism and Inflammation by Branched-Chain Amino Acids and Fatty Acids. Frontiers in Endocrinology, 11, Article No. 617. https://doi.org/10.3389/fendo.2020.00617

[27] Thomas, T., Stefanoni, D., Reisz, J.A., Nemkov, T., Bertolone, L., Francis, R.O., et al. (2020) COVID-19 Infection Results in Alterations of the Kynurenine Pathway and Fatty Acid Metabolism that Correlate with IL-6 Levels and Renal Status. medRxiv. https://doi.org/10.1101/2020.05.14.20102491

[28] Liu, H. and Xia, Y. (2015) Beneficial and Detrimental Role of Adenosine Signaling in Diseases and Therapy. Journal of Applied Physiology, 119, 1173-1182. https://doi.org/10.1152/japplphysiol.00350.2015

[29] Zhang, X., Dash, R.K., Jacobs, E.R., Camara, A.K.S., Clough, A.V. and Audi, S.H. (2018) Integrated Computational Model of the Bioenergetics of Isolated Lung Mitochondria. PLoS ONE, 13, Article ID: e0197921. https://doi.org/10.1371/journal.pone.0197921

[30] Franco, R., Pugliese, A.M., Sun, Y. and Huang, P. (2016) Adenosine A 2B Receptor: From Cell Biology to Human Diseases. Frontiers in Chemistry, 1, 37,

[31] Koukourakis, M.I. and Giatromanolaki, A. (2018) Biology Warburg Effect, Lactate Dehydrogenase and Radio/Chemo-Therapy Efficacy. International Journal of Radiation, 95, 408-426. https://doi.org/10.1080/09553002.2018.1490041

[32] de Oliveira Filho, C.M., Vieceli, T., de Fraga Bassotto, C., da Rosa Barbato, J.P., Garcia, T.S. and Scheffel, R.S. (2021) Organizing Pneumonia: A Late Phase Complication of COVID-19 Responding Dramatically to Corticosteroids. The Brazilian Journal of Infectious Diseases, 25, Article ID: 101541. https://doi.org/10.1016/j.bjid.2021.101541

[33] Pakdemirli, E., Mandalia, U. and Monib, S. (2020) Characteristics of Chest CT Images in Patients With COVID-19 Pneumonia in London, UK. Cureus, 12, Article ID: e10289. https://doi.org/10.7759/cureus.10289

[34] Guan, X., Yao, L., Tan,Y., Shen, Z., Zheng, H., Zhou, H., et al. (2021) Quantitative and Semi-Quantitative CT Assessments of Lung Lesion Burden in COVID-19 Pneumonia. Scientific Reports, 11, Article No. 5148. https://doi.org/10.1038/s41598-021-84561-7

[35] Kwee, T.C. and Kwee, R.M. (2020) Chest CT in COVID-19: What the Radiologist Needs to Know. RadioGraphics, 40, 1848-1865. https://doi.org/10.1148/rg.2020200159

[36] Pinto-Lopes, P., Silva, A.S. and Pimenta, J. (2016) "Non-Resolving Pneumonia": A Case of Cryptogenic Organizing Pneumonia. Porto Biomedical Journal, 1, 191-192. https://doi.org/10.1016/j.pbj.2016.10.004

[37] Balogun, O.O., Fawole, A., Osemwinyen, E. and Balogun, B. (2021) The Placental Buffer Effect and the Pathophysiology of COVID-19: Possibilities for a Guide Aimed at Pregnant and Postpartum Women Considering Praxis: Theory, Clinical and Laboratory Observation. Journal of Infectious Diseases and Epidemiology, 7, Article No. 234. https://doi.org/10.23937/2474-3658/1510234

[38] Fredholm, B.B. (2007) Adenosine, an Endogenous Distress Signal, Modulates Tissue Damage and Repair. Cell Death \& Differentiation, 14, 1315-1323.

https://doi.org/10.1038/sj.cdd.4402132

[39] Bowser, J.L., Lee, J.W., Yuan, X. and Eltzschig, H.K. (2017) The Hypoxia-Adenosine Link during Inflammation. Journal of Applied Physiology, 123, 1303-1320. 
https://doi.org/10.1152/japplphysiol.00101.2017

[40] Escudero, C., Roberts, J.M., Myatt. L. and Feoktistov, I. (2014) Impaired Adenosine-Mediated Angiogenesis in Preeclampsia: Potential Implications for Fetal Programming. Frontiers in Pharmacology, 5, Article No. 134. https://doi.org/10.3389/fphar.2014.00134

[41] Gomes, J.I., Farinha-Ferreira, M., Rei, N., Gonçalves-Ribeiro, J., Ribeiro, J.A., Sebastião, A.M., et al. (2021) Of Adenosine and the Blues: The Adenosinergic System in the Pathophysiology and Treatment of Major Depressive Disorder. Pharmacological Research, 163, Article ID: 105363. https://doi.org/10.1016/j.phrs.2020.105363

[42] Borea, P.A., Gessi, S., Merighi, S., Vincenzi, F. and Varani, K. (2017) Pathological Overproduction: The Bad Side of Adenosine. British Journal of Pharmacology, 174, 1945-1960. https://doi.org/10.1111/bph.13763

[43] Barros D’Elia Zanella, L.G.F.A. and de Lima Galvão, L. (2021) The COVID-19 Burden or Tryptophan Syndrome: Autoimmunity, Immunoparalysis and Tolerance in a Tumorigenic Environment. Journal of Infectious Diseases and Epidemiology, 7, Article No. 195. https://doi.org/10.23937/2474-3658/1510195

[44] D’Elia Zanella, L.G.F. de A.B. (2022) (1) (PDF) The COVID-19 'Bad TryP' Syndrome: NAD/NADH+, Tryptophan-Phenylalanine metabolism and Thermogenesis like Hecatomb-The Hypothesis of Pathophysiology Based on a Compared COVID-19 and Yellow Fever Inflammatory Skeleton. (In Press) https://tinyurl.com/2p9hzz7v

[45] Barros D’Elia Zanella, L.G.F.A. (2021) NEUROCOV/PSYCCOV: Neuropsychiatric Phenomena in COVID-19-Exposing Their Hidden Essence and Warning against Iatrogenesis. Journal of Infectious Diseases and Epidemiology, 7, Article No. 222. https://doi.org/10.23937/2474-3658/1510222

[46] Sauñe, P.M., Bryce-Alberti, M., Portmann-Baracco, A.S. and Accinelli, R.A. (2020) Methylprednisolone Pulse Therapy: An Alternative Management of Severe COVID-19. Respiratory Medicine Case Reports, 31, Article ID: 101221. https://doi.org/10.1016/j.rmcr.2020.101221

[47] Zaitsev, A.A., Golukhova, E.Z., Mamalyga, M.L., Chernov, S.A., Rybka, M.M., Kryukov, E.V., et al. (2020) Efficacy of Methylprednisolone Pulse Therapy in Patients with COVID-19. Clinical Microbiology and Antimicrobial Chemotherapy, 22, 88-91. https://doi.org/10.36488/cmac.2020.2.88-91

[48] Ho, J.C., Ooi, G.C., Mok, T.Y., Chan, J.W., Hung, I., Lam, B., et al. (2003) High-Dose Pulse versus Nonpulse Corticosteroid Regimens in Severe Acute Respiratory Syndrome. American Journal of Respiratory and Critical Care Medicine, 168, 1449-1456. https://doi.org/10.1164/rccm.200306-766OC

[49] Ruiz-Irastorza, G., Pijoan, J.-I., Bereciartua, E., Dunder, S., Dominguez, J., Garcia-Escudero, P., et al. (2020) Second Week Methyl-Prednisolone Pulses Improve Prognosis in Patients with Severe Coronavirus Disease 2019 Pneumonia: An Observational Comparative Study Using Routine Care Data. PLOS ONE, 15, Articke ID: e0239401. https://doi.org/10.1371/journal.pone.0239401 


\section{Abbreviations and Acronyms}

MIS-A Multisystem Inflammatory Syndrome in adults, MIS-c Multisystem Inflammatory Syndrome in child, LDH Lactate dehydrogenase, ADA adenosine, Try L-Tryptophan, 5-HT Serotonin, LCS Long COVID-19 Syndrome, SS Septic Shock, ISC Inflammatory Shock by COVID-19. L-kynurenine (L-kyn). kynurenine monooxygenase (KMO) to form 3-hydroxykynurenine (3-HK). kynureninase (KYNU). quinolinic acid (QUIN) or 2-aminomuconic-6-semialdehyde (AMS). picolinic acid (PICA). 


\section{Appendix}

\section{A1. Diagnostic Criteria for "The Long COVID-19 Syndrome" Based on Author Experience and Literature Review}

1) Serology (points $=0.5$ )

If IgA, IgM, or IgG are positive, confirm considering clinical signs and symptoms. We must consider vaccination. During a pandemic, do not consider the result as false positive.

If the results are negative: it does not exclude acute disease, as the antibody titre may be below the detectable limit by currently available tests.

2) Molecular Exams (points $=0.5$ )

A negative result does not exclude acute infection.

3) Pulmonary image (points $=1.0$ )

Need to perform lung computed tomography. Images showing sequelae as indicated in this article or pattern of Organizing Pneumonia in organization suggest LCS.

4) Clinical History/Anamnesis (points = 1.0)

Previous flu-like symptoms with more than 14 days of evolution. History of pneumonia or hospital admission in the pandemic period. We should be aware that many patients were hospitalized with a diagnosis of bacterial pneumonia due to the presence of consolidations in the lungs and neutrophilia in the blood. But this is the natural evolution of the image in COVID-19.

5) Elevated serum LDH and Lactate (points $=2.0$ )

They are elevated due to the diversion of aerobic respiration towards the axis of oxidative stress and inflammation.

6) Acute lowering of the level of consciousness (points =2.0)

$\mathrm{Kt}$ is a prevalent sign, especially in the elderly, with cerebrospinal fluid without alterations and excluding stroke.

7) PCR and ESR generally high (points $=2.0$ )

These parameters are usually elevated due to systemic inflammation.

8) Chronic pain or abdominal or neuropsychiatric symptoms (points $=2.0$ )

Signals and symptoms initiated after hospitalization or pneumonia in the pandemic period.

Common symptoms are diffusely distributed pain, tenesmus, or diarrhoea with blood or mucus: Depression or anxiety, psychomotor agitation.

9) Consumptive Syndrome (points $=3.0$ )

Very present mainly in the elderly due to deviation of try metabolism and inflammation via oxidative stress.

10) Lymopenia or pancytopenia, relative monocytosis, basophilia, thrombocytosis with phases of thrombocytopenia, eosinophilia (points 3 )

Important data should be observed in the blood count, as they may be associated with acute or previous COVID-19, whose maintenance suggests LCS.

11) Urine exam (points $=3.0$ )

Leukocyturia, proteinuria varying in ascending with improvement after 
treatment, excluding previous kidney damage.

Presence of cylindric, epithelial cells

12) Bradycardia (points $=3.0$ )

Bradycardia in situations of need for tachycardia may suggest metabolic exhaustion and myocardial damage.

\section{A Score Greater Than or Equal to 4.0 Suggests LCS.}

\section{A2. Prescription for the Moderated, Severe and Critical Patient}

1) Protein-rich diet

2) Intravenous $10 \%$ Glucose Serum adjusted according to capillary blood glucose.

3) Insulin pump $-0.9 \%$ saline + regular Fast Insulin. Intravenous with adjustment according to capillary blood glucose.

4) Intravenous heparin pump with adjustment according to APTT.

5) Vitamin B Complex 2 ampoules 1 time a day.

6) Fat-soluble and water-soluble vitamins intravenously once a day (including 1 to 2 grams/day of Vitamin C and thiamine $1200 \mathrm{mg}$ daily for the first 3 days of admission.

7) $8.4 \%$ Intravenous sodium bicarbonate

8) Sodium bicarbonate $8.4 \% 70 \mathrm{~mL}+$ saline $0.9 \%$ intravenously, in continuous infusion pump for $8 / 8 \mathrm{~h}$.

9) \#Maintain $\mathrm{pH}$ greater than or equal to 7.25 .

10) Heparin pump or prophylaxis ${ }^{\star}$

11) Acetylsalicylic acid $100 \mathrm{mg}$ once a day.

12) $\mathrm{N}$-acetyl cysteine $600 \mathrm{mg}$ once a day.

13) Folic acid $5 \mathrm{mg} /$ day.

14) Taurine 2 grams once a day.

15) Citalopram $40 \mathrm{mg}$ once a day.

16) Ondansetron $8 \mathrm{mg}$ of $12 / 12 \mathrm{~h}$.

17) Folic acid $5 \mathrm{mg} /$ day

18) Atorvastatin $80 \mathrm{mg} /$ day.

19) Levodopa+ Benserazide 200/50mg once a day.

20) Dipyrone 1 gram of $6 / 6 \mathrm{~h}$ intravenously or Paracetamol $500 \mathrm{mg}$ of $8 / 8 \mathrm{~h}$ if pain or fever.

21) Albumin 20\% 2 initial vials followed by 1 vial every 4 hours until reaching serum albumin of $3.5 \mathrm{~g} / \mathrm{dL}$.

22) Glucose $50 \% 4$ ampoules intravenous capillary blood glucose $<70 \mathrm{~g} / \mathrm{dL}$.

23) Continuous monitoring of blood pressure, Oximetry $\left(\mathrm{O}_{2}\right.$ Saturation), respiratory rate.

24) Capillary blood glucose $1 / 1 \mathrm{~h}$ or $2 / 2 \mathrm{~h}$.

25) Vesical indwelling probe and diuresis quantification.

26) Keep blood glucose between 120 and $200 \mathrm{~g} / \mathrm{dL}$.

27) $\mathrm{Keep} \mathrm{FiO}_{2} 40 \%$ even if the patient is weaning. Maintain protective ventilation and adjust the ventilator to the patient, titrating the need for PEEP, tidal 
volume to maintain a $\mathrm{pH}$ greater than or equal to 7.25 and $\mathrm{pCO}_{2}$ as close to physiological as possible. Avoid permissive hypercapnia.

${ }^{*}$ Risk factors for bleeding that contraindicate pharmacologic thromboprophylaxis. Active bleeding 6. Acute stroke 2. Acquired bleeding disorders 7. Thrombocytopenia $<25 \times 109 / \mathrm{L}\left({ }^{*}\right) 3$. Concurrent use of anticoagulants 8 . Uncontrolled systolic hypertension 4 . Lumbar puncture/epidural/spinal anesthesia expected within the next $12 \mathrm{~h} 9$. Untreated inherited bleeding disorders 5. Lumbar puncture/epidural/spinal anesthesia within the previous $4 \mathrm{~h}$.

\section{A3. Pulse of Methylprednisolone [14] [15] [43] [44] [45] [46]}

It is indicated for both non-shock and shock associated LCS patients.

For moderated, severe or critical disease

a) Author's Indication:

METHYLPREDNIOLONE 1000 mg or 500 MG INTRA VENOUS FOR 3 DAYS WITH UNASSOCIATED WITH.

b) Iranian protocol (Maryan Edalatfard et al.)

METHYLPREDNISOLONE 250 MG FOR 3 DAYS AS PERFORMED BY THE IRANIAN PROTOCOL DURING THE ACUTE PHASE OF THE DISEASE.

Perform Ivermectin $6 \mathrm{mg} 2$ tablets in a single dose as prophylaxis for disseminated strongyloidiasis.

Associate antimicrobial if there are positive blood, urine or bronchoalveolar lavage cultures

Antimicrobial

It is not mandatory initially, but as there is a high possibility of bacterial translocation in the LCS, start Ceftriaxone $1 \mathrm{~g}$ every 12 hours if the medical evaluation shows a possibility of over-aggregated bacterial infection.

[47] [48] [49]

Decreasing Methylprednisolone doses:

$80 \mathrm{mg}$ for 3 days

$60 \mathrm{mg}$ for 4 days

Totalling 14 days of treatment dose.

The dose of 250 may need to be repeated on day 14 and day 21. Maintain a 20 mg dose of Prednisolone for hospital discharge.

For patients without the need for hospital admission:

Prednisolone $240 \mathrm{mg}$ for 3 days.

Moving on to the weaning phase:

Prednisolone $160 \mathrm{mg}$ for 3 days.

Prednisolone $80 \mathrm{mg}$ for 2 days.

Prednisolone $40 \mathrm{mg}$ for 2 days.

Prednisolone $10 \mathrm{mg}$ for 2 days.

After 7 days: Prednisolone $10 \mathrm{mg}$.

1) Citalopram $40 \mathrm{mg}$ once daily for 30 days. (reassess the need to continue or 
not)

2) Atorvastatin $80 \mathrm{mg}$ for 15 days and lowering the dose to $40 \mathrm{mg}$ a day after completing 60 days. (order exams and reassess the need to continue or not.

3) Taurine $1500 \mathrm{mg}$ daily for 30 days.

4) Vitamin B complex containing B3: 1 tablet once daily.

5) Acetyl salicylic acid $100 \mathrm{mg}$ once daily for 15 days. continue for 2 months doing $100 \mathrm{mg} 3$ times a week.

6) N-Acetylcysteine $600 \mathrm{mg}$ once daily for 15 days

7) Tryptophan $1000 \mathrm{mg}$. Start on the fourth day of treatment initiation. Take 1 tablet daily for 7 days, discontinue for 7 days and resume treatment. Follow this sequence for 30 days and reassess the need to continue with the drug.

8) Clopidogrel $75 \mathrm{mg}$. Take 1 tablet daily (if associated cardiovascular risk), reassess after 30 days.

9) Caffeine $1000 \mathrm{mg}$. Take 1 tablet early if the patient has symptoms of anxiety or akathisia. (Adenosine antagonist).

Always, if possible, start treatment before the cytokine storm, preferably between D6 and D8 of the onset of symptoms with C-reactive protein (CRP) less than or equal to $10 \mathrm{mg} / \mathrm{dL}$, that is, before the cytokine storm, as the desired effect the storm blocks.

Prescribe Ivermectin $6 \mathrm{mg} 2$ tablets to be taken as a single dose.

Inform that if there is a worsening or if saturation drops to $\mathrm{O}_{2}$ Saturation $\left(\mathrm{SatO}_{2}\right)$ value less than or equal to 95\%, supplementation should be started at home or the hospital.

Complete rest for 15 days and resume small activities without forcing $\mathrm{O}_{2}$ demand to perform physical activities.

Request return within 5 days for outpatient reassessment.

\section{A4. Exams for Clinical Follow-Up}

a. Admission

-COMPLETE CBC WITH PLATELETS

DHL

-LACTATe

UREA

\section{-CREATININE}

-Sodium, potassium, ionic calcium, magnesium

urine screening

-Ferritin

-Total proteins and fractions

-ASL/ALT, Gamma-GT, Alkaline Phosphatase, Total Bilirubins

-TSH, Free T4, parathyroid hormone.

-Total cholesterol and fractions, triglycerides

-Ammonia

Blood 
-Arterial blood gases

-Computerized tomography of the chest. Perform abdomen if necessary

-ELECTROCARDIOGRAM

-CORTISOL

-Coagulogram

-Fibrinogen

-IRON PROFILE

-Vitamin Profile

-C-reactive protein

-CPK

b. clinical follow-up

-Complete blood count WITH PLATELETS

-DHL

-LACTATe

-UREA

-CREATININE

-Sodium, potassium, ionic calcium, magnesium

-Urine screening (daily for the first 5 days and then move to twice a week)

C-REACTIVE PROTEIN

c. Sporadic

-D-Dimer, CPK, CKMB, Troponin only if necessary.

-RHEUMATOLOGICAL PROFILE: if there is a need or a chance of a vasculitic component.

\section{A5. Ventilator Weaning in the Case of an Intubated Patient}

a) Awakening daily.

b) If lower $\mathrm{FiO}_{2}$ is indicated, keep at least $\mathrm{FiO}_{2}=40 \%$ (randomized trials). Lowering $\mathrm{FiO}_{2}$ too much causes a shift from inflammation to oxidative stress. It is important that clinical trials are carried out to verify that $\mathrm{FIO}_{2}$ does not cause lung damage, much less falls short of what should be provided to the patient during weaning. Hypoxia causes agitation due to the toxic products of Kynurenine. 